\title{
Ruralidad, paradojas y tensiones asociadas a la movilización del pueblo Mapuche en Pulmarí (Neuquén, Argentina) ${ }^{1}$
}

\author{
Sebastián Valverde
}

CONICET/FFyL-UBA sebavalverde@yahoo.com.ar

\section{Gabriel Stecher}

Universidad Nacional del Comahue gabrielstecher@gmail.com

\begin{abstract}
Resumen: La región de Pulmarí, en el Departamento Aluminé, en el sur de la Argentina (Provincia de Neuquén) se caracteriza por una destacada presencia del pueblo indígena Mapuche, que ha protagonizado intensas movilizaciones desde la década de 1990 por su territorio ancestral y frente al avance de diferentes agentes estatales y privados. En contraste con la tendencia que afecta a otras poblaciones, en la región se vienen dando procesos de "territorialización" de estas familias indígenas y desaceleración de las históricas migraciones rural - urbanas, lo que conlleva profundas redefiniciones y reconfiguraciones de estos ámbitos rurales -tendencias explicables a partir de la aplicación de diferentes políticas y planes sociales-.

El objetivo principal es analizar la dinámica de las fuentes de ingresos de estos pequeños productores rurales Mapuche, atendiendo a estas particularidades y vinculándolas con el contexto de conflictividad y disputas entre los diferentes sectores que caracteriza a la región desde hace años.
\end{abstract}

Palabras clave: pequeños productores rurales; políticas públicas; Mapuche; Provincia de Neuquén; Pulmarí.

\section{Rurality, paradoxes and tensions associated to the Mapuche people movement in Pulmarí (Neuquén, Argentina)}

The area of Pulmarí, in the district of Aluminé, in the South of Argentina (Province of Neuquén) is characterized by a notorious presence of Mapuche native people, who have been involved in intense movements since the 90s, claiming for their ancestral territories and facing the advance of different public and private agents. As opposed to the trend affecting other peoples, there have been "territorialization" processes in the area in relation to these indigenous families and de-acceleration of the historical rural-urban migrations, which implies deep redefinitions and reconfigurations of these rural environments. Such trends are due to the application of different social policies and plans. The main objective is to explore the dynamics of the income sources of these small Mapuche rural producers, taking into account their special characteristics and linking them to the 
context of conflict and dispute among the different sectors that have characterized this area for years.

Keywords: Small rural producers; public policies; Mapuche; Province of Neuquén; Pulmarí.

\section{Ruralidade, paradoxos e tensões associadas com a mobilização dos Mapuche no Pulmarí (Neuquén, Argentina)}

Resumo: A região Pulmarí, Departamento Aluminé, no sul da Argentina (Província de Neuquén) é caracterizada por uma forte presença do indígena Mapuche, que já atuou intensos protestos da década de 1990 para o seu território ancestral e contra o progresso do estado e vários atores privados. Em contraste com a tendência afetando as populações de outros países da região têm vindo a evoluir processos “territorialização" das famílias indígenas e desaceleração histórica migração rural - urbana, levando a profundas redefinições e reconfigurações destas áreas rurais, as tendências explicáveis a partir da aplicação de diferentes planos políticos e sociais. O objetivo principal é analisar a dinâmica de fontes de renda desses pequenos agricultores rurais Mapuche, considerar essas particularidades e sua ligação com o contexto de conflitos e disputas entre os diferentes setores que caracteriza a região por anos.

Palavras-chave: pequenos agricultores, políticas públicas; Mapuche, província de Neuquén, Pulmarí.

\section{Mapa No 1: Provincia de Neuquén}

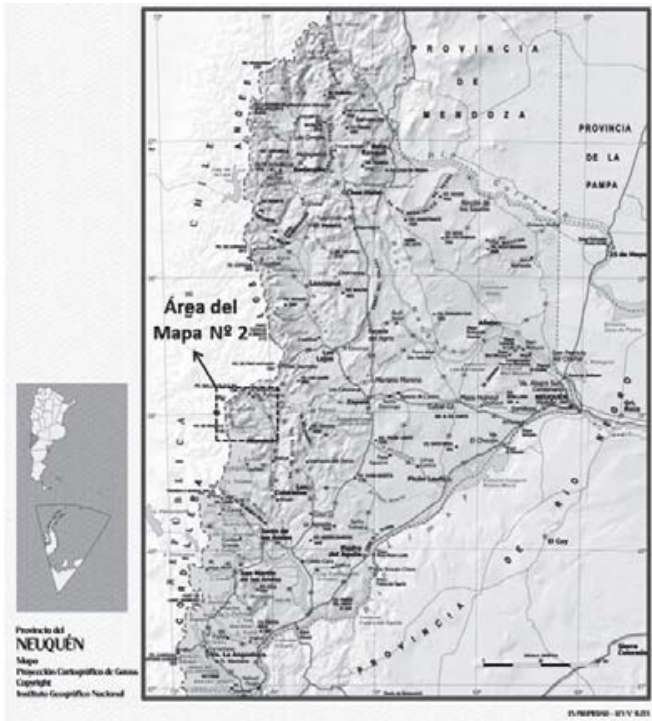

Fuente: Instituto Geográfico Nacional 


\section{Mapa No 2: Área de la Corporación Interestadual Pulmarí (CIP)}

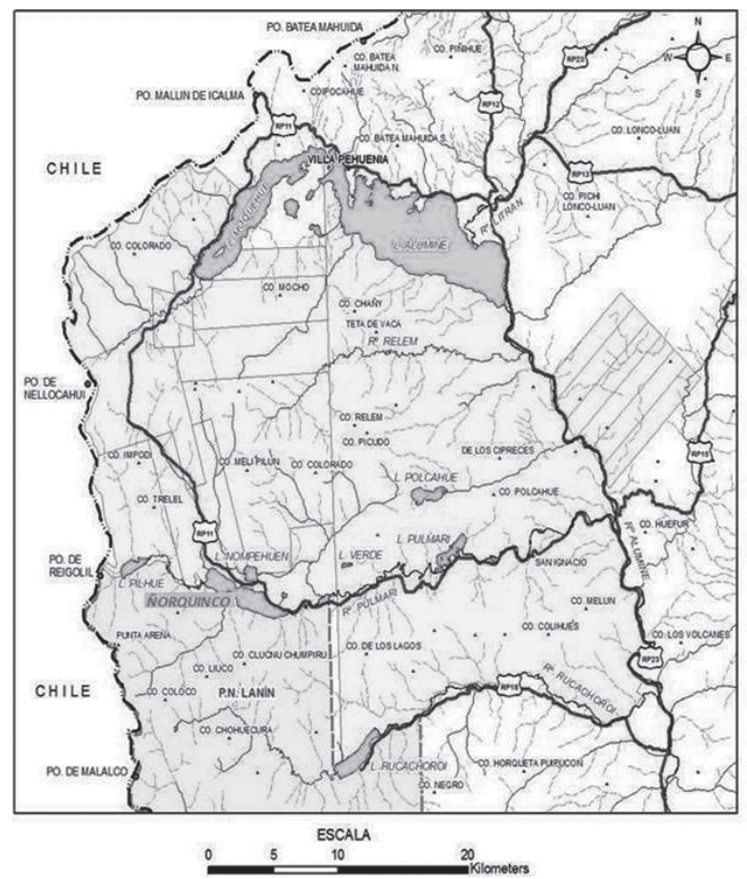

Fuente: Elaborado por Eduardo R. García, Laboratorio de Información Geográfica Forestal. Coordinación de Política Forestal, Prov. del Neuquén.

\section{Introducción}

Cuando tomamos conocimiento de la convocatoria del dossier de la "Revista Polis" que tiene como "lente de aproximación” la temática "Ruralidad y campesinado ¿categorías en extinción o realidades en proceso de transformación?” vimos con beneplácito como la región de Pulmarí, en la Provincia de Neuquén en el sur Argentino -en la que trabajamos desde hace varios años- era sumamente pertinente a este tema (ver Mapas $\mathrm{N}^{\circ} 1$ y 2). Esto renovó nuestro interés por abordar las particularidades de su población indígena rural, donde a contrapelo de la tendencia general, la zona no tiende a desruralizarse sino que -en algún sentido- ocurre lo contrario, advirtiéndose profundas transformaciones (y causas sumamente específicas que explican dichas particularidades). Por eso uno de los desafíos que nos proponemos es el de desentrañar las características de la población indígena Mapuche ${ }^{2}$ del área de Pulmarí, y a la vez contribuir, a partir de este caso específico, a los debates actuales en torno a la ruralidad en la sociedad contemporánea. 
En efecto, esta región se caracteriza por una destacada presencia de este pueblo originario y a la vez, una gran conflictividad asociada al acceso a su territorio ancestral.

Desde fines de la década de 1980, la región se encuentra administrada por la "Corporación Interestadual Pulmarí” (CIP), ente interjuridiccional conformado por tierras de la Nación y la Provincia- que surgió con el (supuesto) fin de mejorar las condiciones de vida de estas comunidades originarias. Pero muy lejos de los propósitos explícitos, desde su conformación las políticas de este organismo tendieron a desmejorar cada vez más las condiciones de vida de las familias indígenas, a causa de la falta de tierras, las restricciones a sus actividades productivas (muchas no sólo de un valor económico sino también cultural) y la falta de una real participación indígena en el directorio de la $\mathrm{CIP}^{3}$. Malestar que finalmente derivó en una intensa movilización indígena que tuvo lugar entre los años 1995-1996 -con profundas consecuencias- transformándose el denominado "conflicto de Pulmarí” en emblemático de las movilizaciones de los pueblos originarios en las últimas décadas, en Argentina en general y en particular en relación al pueblo Mapuche.

Esta zona posee una gran complejidad social, ya que en el área administrada por la CIP se asientan, además de seis comunidades del pueblo indígena Mapuche (Hiengheihual, Aigo, Puel, Catalán, Currumil y Ñorquinco), los denominados "Pobladores de Ley", que son habitantes rurales asentados con anterioridad a la conformación de la CIP, por ello fueron reconocidos por la ley de creación de la misma (Corporación Interestadual Pulmarí 2012). No obstante, su situación de tenencia de la tierra es precaria (sin seguridad jurídica).

A esto le debemos sumar los "Concesionarios", que constituyen particulares a quienes la CIP ha otorgado concesiones con distintos fines (ganaderas, agrícolas, apícolas, turísticas, etc.) por plazos determinados. En algunos casos, en especial las más grandes que conforman verdaderas empresas privadas, suelen mantener situaciones de gran conflictividad con el pueblo Mapuche, por los ámbitos territoriales.

También debemos considerar como otro actor, al Ejército Argentino ${ }^{4}$ quien posee en este ámbito su campo de entrenamiento y maniobras; con su consecuente carga simbólica -tanto por la trágica década de 1970 y principios de los '80 en nuestro país, como por la avanzada militar contra el pueblo Mapuche a fines del Siglo XIX)-.

Cabe destacar (si bien luego nos referiremos con mayor detalle a este aspecto) que las principales actividades económicas de la región son la ganadería, la forestación y el turismo. La primera de ellas es la de mayor tradición social, practicada por grandes, medianos y pequeños productores -entre estos últimos los integrantes de las comunidades indígenas-. En efecto, los miembros de estas comunidades son pequeños crianceros, principalmente de ovinos y caprinos, y en segundo lugar de bovinos (Stecher 2011). 
En lo que respecta a la trascendencia en esta zona de la actividad forestal, esto explica que otros actores con presencia en el área Pulmarí, son empresas forestales ya sea estatales (o mixtas) como la Corporación Forestal del Neuquén (CORFONE S. A.) -quien además de campos propios posee concesiones dentro de la CIP- o capitales privados ${ }^{5}$. También se ha sumado a este territorio empresas que denominamos "neo rurales" (Stecher y Laclau 2010a y 2010b) tales como aquellas cuya principal actividad se relaciona con el sector energético pero también han diversificado su accionar, efectuando en esta zona inversiones forestales.

Como es factible observar, existe una gran diversidad en términos socioculturales, económicos y políticos, entre -y dentro- de los sectores que participan y disputan esta área administrada por la CIP. Esto implica un contexto de gran complejidad social, fruto precisamente de la superposición de diferentes jurisdicciones sobre los territorios, con diversas normas, relaciones político-administrativas y posicionamientos políticos, los cuales a su vez despliegan diferentes estrategias que se han ido modificando a lo largo del tiempo.

Este entramado, contribuye a explicar por qué desde las movilizaciones de la década de 1990, la región se caracteriza por los elevados niveles de conflictividad aún irresueltos, la judicialización de la movilización indígena, así como las agudas disputas entre los diferentes -y variados- sectores que interactúan en la administración de la CIP y en estos espacios territoriales.

Otro aspecto que aquí nos interesa enfatizar es que una de las consecuencias del "conflicto de Pulmarí” de aquellos años y de los litigios posteriores, ha sido la "visibilización” de la población indígena y rural (en sus aspectos conflictivos, productivos, situaciones de pobreza, etc.), en contraposición con las tendencias hegemónicas a la negación e invisibilización -pero también en algunos casos folklorización-. De allí, que en la región se instrumenten diversas políticas sociales, planes y proyectos de desarrollo que tienen como destinataria a la población indígena, con situaciones sumamente variadas en lo que respecta a los niveles de participación de la misma en su diseño e implementación.

Presentados estos antecedentes, deseamos señalar que una de las tendencias que caracteriza a la región -en abierto contraste con la mayor parte de las zonas cercanas de Patagonia, otras de la Argentina y de América Latina- es la presencia y crecimiento de la población rural, diversos procesos territorialización o re-territorialización y desaceleración de las históricas migraciones rural - urbanas. A su vez, las familias indígenas desarrollan múltiples actividades no agrícolas, donde la aplicación de políticas públicas resulta una variable fundamental, con profundas redefiniciones y reconfiguraciones de estos ámbitos rurales.

Así, el objetivo central del presente ensayo es analizar la dinámica de las unidades domésticas de pequeños productores rurales que integran 
las comunidades Mapuche asentadas en la CIP, atendiendo a las particularidades ya descriptas y vinculándolas con el contexto de conflictividad y disputas entre los diferentes sectores que caracteriza a la región desde hace años. Finalmente, nos proponemos que el estudio de este caso -por cierto emblemático de la lucha de sectores rurales indígenas en Argentina- redunde en un aporte al debate inherente a la temática de lo rural en la actualidad, con las profundas transformaciones, redefiniciones y a la vez problematizaciones que como investigadores debemos efectuar.

Para el desarrollo de este ensayo hemos empleado diferentes fuentes documentales, y realizado también entrevistas entre los años 2007 y 2012, a integrantes y dirigentes de las comunidades Mapuche, así como otros sectores involucrados. Uno de los autores de este trabajo -Dr. Sebastián Valverde- como antropólogo social investiga desde hace años la conflictividad territorial del pueblo Mapuche en relación a los procesos de actualización étnico-identitaria, las transformaciones socioeconómicas regionales y el desarrollo de los movimientos indígenas. El otro autor -Dr. Gabriel Stecher- como Técnico Forestal (como formación de grado) y en Estudios Sociales Agrarios (de posgrado) se ha desempeñado en diferentes proyectos de intervención en la región con las poblaciones indígenas y viene investigando sobre estas dinámicas, por lo que parte de lo aquí abordado surge de dichas experiencias.

Cabe señalar que efectuamos un recorte temporal hasta el año 2009, ya que una parte importante de los datos surgen de una serie de relevamientos efectuados en tal año. Por otro lado, en el año 2010 se implementó la denominada “Asignación Universal por Hijo" -que implica una prestación a amplios sectores sociales- y resulta de gran impacto en las comunidades, lo que requeriría de un estudio específico y más actualizado de mayor profundidad (actualmente en curso). No obstante, el recorte en el año 2009 resulta además sumamente pertinente, ya que en ese año se han desarrollado intensos conflictos en la zona, plenamente contextualizables a partir de dichos datos.

Iniciamos este trabajo con una presentación de los diferentes lineamientos teóricos. Luego presentaremos las características ambientales y sociales de esta región y de las comunidades indígenas asentadas en la misma, así como los antecedentes del conflicto de los años 1995-1996. Seguidamente, daremos cuenta de las diferentes fuentes de ingreso, la retención de la población rural y los efectos de las políticas públicas en las actividades tradicionales.

\section{Aspectos teóricos y metodológicos}

Retomaremos las caracterizaciones que se vienen efectuando inherentes al medio rural latinoamericano en las últimas décadas (Giarraca 2001; Teubal 2001; Rubio 2002; Grammont 2008) que implican profundas transformaciones. 
Parte de las tendencias que aquí nos interesa remarcar -ya que involucran a pequeños productores rurales Mapuche en Norpatagonia- se asocian con la creciente pluriactividad y diversificación entre diferentes fuentes de ingreso de las unidades domésticas -proceso denominado comúnmente "desagrarización”- (Rubio 2002), así como la desaceleración (o prácticamente inexistencia) de migraciones definitivas a las ciudades, debido a la falta de empleo y las nuevas condiciones precarias del mercado de trabajo (Grammont 2008).

No obstante, las restricciones para la venta en el mercado interno (con la consiguiente exclusión y caída de sus ingresos) (Rubio 2002) como ocurre con otros sectores rurales, en este caso no afecta a este segmento de pequeños productores rurales Mapuche, ya que hace varias décadas no comercializaban sus producciones masivamente. De hecho, esta ha sido una de las causas de las migraciones rural-urbanas, que se dieron generaciones atrás.

En el caso de los pequeños productores Mapuche -como veremos-, la tendencia al crecimiento demográfico de las familias en los mismos ámbitos territoriales en que se desarrollan las actividades de subsistencia, lleva crecientemente a diversificar los ingresos, siendo esta una de las causas fundamentales de la denominada "pluriactividad" que caracteriza crecientemente a estos productores rurales (Grammont 2008). A esto se le suma el peso de los planes sociales que vienen siendo instrumentados en la provincia como forma de paliar las situaciones de pobreza.

Otros de los lineamientos teóricos que retomamos, están asociados a diferentes autores (Wolf 1993; Rosberry 1991) que vinculan lo global, con lo local considerando para ello -tal como ha planteado este último autor para los campesinos en Venezuela- diferentes niveles de análisis, que implican visualizar la forma en que dinámicas más generales han incidido en sujetos particulares y cómo los efectos de tales interrelaciones no resultan uniformes, sino sumamente diferenciales.

El otro aspecto que retomaremos de esta propuesta, es el carácter histórico, que permite precisamente considerar la forma en que a lo largo del tiempo se ha dado la vinculación entre lo macro y lo micro (visto como un continuum, no como categorías dicotómicas), y la forma en que los sujetos particulares han buscado modificar dicha realidad. En palabras de Rosberry, es necesario “(...) prestar especial atención a las presiones externas y las respuestas internas a lo largo del tiempo” (1991:167).

El hecho de efectuar esta interrelación entre aspectos generales y locales en términos procesuales, permite "desnaturalizar" las realidades que observamos en los ámbitos de estudio. Favorece -parafraseando a este autor- "(...) tomar conciencia de la posibilidad de que las características de la vida que parecen más tradicionales o habituales sean resultado de imposiciones, respuestas o acomodos que tuvieron lugar en el pasado" (Rosberry 1991:167). 
La pregunta que surge naturalmente es; ¿cuál es el contexto temporal y espacial para considerar como marco de dichas dinámicas? Aquí retomamos los planteos del enfoque denominado "histórico-regional" 6 desarrollado desde la disciplina histórica, que viene teniendo una importante influencia en las investigaciones acerca de la región de Norpatagonia de otras disciplinas como la Antropología, la Arqueología, la Geografía, la Sociología, etc. ${ }^{7}$. Desde estas lecturas alternativas a las concepciones positivistas, se desecha la definición “apriorística” del objeto de estudio, tanto en términos espaciales como temporales, ya que, en efecto, la región se constituye “(...) a partir de las interacciones sociales que la definen como tal en el espacio y en el tiempo, dejando de lado cualquier delimitación previa que pretenda concebirla como una totalidad preexistente" (Bandieri 2001:6). Así, la región entonces se define (y forma parte) del proceso de investigación mismo.

Desde esta perspectiva es que visualizaremos el rol del Estado Neuquino, la generación de importantes recursos como la actividad hidrocarburífera con la consiguiente intervención en los ámbitos rurales, y cómo estas políticas inciden en la dinámica de las unidades domésticas. Esto contribuye a dar cuenta de la pluriactividad, la creciente diversificación de ingresos y a la interrelación entre las actividades agrícolas con los planes sociales (monetarios y no monetarios).

A su vez, esto se relaciona con los cambios a lo largo del tiempo, la influencia del conflicto de 1995-1996, las políticas que buscan paliar la pobreza y el desempleo, pero que -paradójicamente- contribuyen a territorializar a las poblaciones indígenas y rurales.

Indudablemente, esto también implica una compleja estrategia metodológica para el abordaje de esta problemática, ya que muchas dinámicas generales es necesario conocerlas a partir de estadísticas, así como diversas fuentes documentales, etc. Pero otra fuente metodológica fundamental, sigue siendo el método etnográfico característico de la antropología. Precisamente las entrevistas en profundidad posibilitan en más de una ocasión visualizar claramente cómo dinámicas generales han afectado a sujetos particulares.

\section{Las características del Departamento Aluminé}

La Provincia del Neuquén se localiza en el noroeste de la Patagonia, recostada sobre la Cordillera de los Andes. Posee una superficie de 94.078 $\mathrm{km}^{2}$ y está dividida administrativamente en 16 departamentos. Limita al Norte con la provincia de Mendoza, al Sur con la provincia de Río Negro, al oeste con la cordillera de los Andes (límite natural que la separa de la República de Chile) y al Este con Río Negro y La Pampa.

El Departamento en que nos centraremos, el de Aluminé, se encuentra localizado en el centro-oeste de la provincia de Neuquén y limita con el 
vecino país de Chile a lo largo de la Cordillera de los Andes, ocupando una superficie de $4.660 \mathrm{~km}^{2}$ (lo que equivale al 5\% del total provincial). La localidad cabecera lleva el mismo nombre que el distrito, asentándose a 317 km de la Capital provincial, la ciudad de Neuquén (que reúne a casi la mitad de la población de la Provincia) (INDEC 2001). La otra localidad es Villa Pehuenia ${ }^{8}$.

De acuerdo a datos del censo del año 2001, el conjunto del Departamento de Aluminé contaba por aquel entonces con una población de 6.308 habitantes, siendo un 54,9\% de población urbana y 45,1\% rural, distribución que contrastaba marcadamente con el total provincial (88,6\% para el primer caso y 11,4\% para el segundo) (INDEC 2001). En el registro anterior (año 1991), había presentado una cifra de 4.946 habitantes, lo que constituye un incremento del 28,7\%. Los datos del último censo (del año 2010) dan cuenta de 8.306 habitantes, lo que implica un crecimiento del $31,7 \%$ respecto del año 2001, siendo este incremento prácticamente el doble comparado con la media provincial (16,3\%) (INDEC 2010). Esta suba puede ser explicada a partir de migraciones internas de otras zonas de la provincia y del país (Stecher y Berenger 2009).

La situación socioeconómica del Departamento de Aluminé presenta características signadas por la importancia demográfica de la población rural e indígena (Stecher 2011). En base a datos del año 2001 (ya que en este indicador los más recientes del año 2010 aún no se encuentran disponibles), los hogares urbanos con Necesidades Básicas Insatisfechas (NBI) para la localidad de Aluminé es de 13,7\%, levemente inferior al total provincial que llega al 15,4\%. Estos valores no parecen -en sí- ser críticos, pero si lo consideramos exclusivamente para el área rural, ascienden a un 35,5\%. Al considerar la población indígena rural, la proporción de hogares que poseen esta situación de privación alcanza un alarmante $47,1 \%{ }^{9}$.

Las características naturales que presenta el área, permiten comprender las causas de los múltiples intereses en pugna, por parte de los diferentes agentes intervinientes como empresas y el propio Estado (Nawel et al. 2004). El origen glaciario de sus lagos, hace que sea muy rica en recursos hídricos por lo cual sean muy valorados para la actividad turística. El paisaje boscoso -en especial en la zona oeste montañosa del Departamento- representa una gran riqueza en términos paisajísticos, en cuanto a la biodiversidad y a la vez para el desarrollo forestal. De hecho los suelos derivados de cenizas volcánicas los hacen aptos para la actividad forestal, lo que contribuye a explicar -entre otros factores- que el Departamento Aluminé sea el de mayor superficie forestada de la Provincia (Stecher 2011; Stecher y Valverde 2012) $)^{10}$.

Cabe destacar, que algunas especies poseen además un importante valor cultural para el pueblo Mapuche, como es el caso de la Araucaria o Pehuen (Araucaria araucana) y su fruto “el piñón” parte de su base alimentaria. 
La otra actividad asociada al ambiente de la región, es la ganadería, favorecida por la presencia de mallines. Es practicada por grandes, medianos y pequeños productores (entre estos últimos los integrantes de las comunidades indígenas) siendo muy amplia la heterogeneidad dentro de cada uno de estos grupos y entre sí. Los pobladores Mapuche son pequeños crianceros, principalmente de ovinos y caprinos, y en segundo lugar de bovinos. Esta representa una de sus principales fuentes de ingreso (junto con los planes sociales del Estado y en menor medida, trabajos asalariados temporarios o permanentes). No obstante, en los últimos años la actividad ganadera presenta serias dificultades dado el avanzado grado de erosión de las tierras que ocupan. Por ello, como las comunidades tienen limitaciones para acceder al territorio ancestral, se produce el desequilibrio que provoca este sobrepastoreo, generándose un fuerte proceso de degradación y consecuente pérdida productiva de los suelos (Stecher 2011). Pero además parte de los terrenos más propicios para esta actividad, se encuentran ocupados por los concesionarios y empresas -como CORFONE- lo que limita la capacidad de pastoreo de los ya de por sí escasos campos de las comunidades, con los consiguientes conflictos que ello implica (Nawel et al. 2004; Stecher 2011; Stecher y Valverde 2012).

\section{El conflicto de los años 1995-1996 y sus consecuencias}

Una vez presentados los antecedentes de la región, debemos mencionar brevemente el conflicto de los años 1995-1996, dado los profundos efectos que han tenido.

En el año 1984 -a pocos meses de reinstaurarse la democracia- durante una visita a la Provincia de Neuquén el entonces Presidente Raúl Alfonsín, afirmó: “Ahora, Pulmarí es para los Mapuches” (Ñancucheo 1998:20). Así es como se originó la CIP, organismo que surge de la fusión de tierras fiscales provinciales (45.000 hectáreas) y nacionales (antigua estancia Pulmarí, que fue expropiada a la familia Miles de capitales ingleses a fines de los años ' 40 y cedida al Ejército Argentino, con un total de 67.900 hectáreas), asentándose por aquel entonces, en su jurisdicción, seis comunidades Mapuche: Aigo, Puel, Catalán, Currumil, Hiengheihual y Ñorquinco (de las cuales la CIP, sólo reconocía a las cuatro primeras, no así a las dos últimas de más reciente reorganización).

No se equivocó el por entonces primer mandatario, al proclamar que con la puesta en funciones de la CIP (en el año $1988^{11}$ ) los cambios no se harían esperar. No obstante, como hemos señalado precedentemente, los mismos fueron en sentido opuesto a lo prometido. Con el correr del tiempo, se fue dando un deterioro aún mayor en las condiciones de vida de las familias Mapuche, a causa de la falta de tierras y de la escalada de medidas restrictivas relacionadas con sus actividades productivas. En términos económicos, la imposición de tasas abusivas de pastaje (cobradas en animales o especies), la prohibición de recolectar y vender piñones (fruto del árbol “pehuén” o “araucaria”) y las limitaciones a la recolección de leña, fueron 
afectando la supervivencia de las familias (Radovich 2000; Carrasco y Briones 1996; Valverde 2009; Delrio et al. 2010). A la vez, fue sumamente restringida la entrega de tierras a indígenas, contrastando con las generosas concesiones efectuadas a particulares. También creaba importantes conflictos la presencia de un sólo representante indígena (en un directorio compuesto por un total de ocho miembros) al que, además, la Provincia de Neuquén se adjudicó el derecho a designar, lo que acrecentó la tensión. Finalmente, persistía como reclamo el reconocimiento de las comunidades Ñorquinco y Hiengheihual (Salazar), de reciente reorganización, como comunidades hacia adentro de la CIP, que este Ente se negaba a reconocer como interlocutores válidos.

Este malestar, derivó en una intensa movilización que tuvo lugar en el año 1995 con la ocupación de la sede de la CIP -por parte de integrantes de la Confederación Mapuche Neuquina y las comunidades- la toma de campos en litigio, diversas declaraciones públicas y acciones de solidaridad de diferentes sectores sociales. La respuesta del gobierno no se hizo esperar, ya que acusó a los dirigentes Mapuche del delito de "usurpación de tierras”, a partir de la apertura de diversas causas. Esta judicialización de la protesta indígena fue acompañada por una campaña mediática -reflejada en diferentes discursos y notas periodísticas- que enfatizaba las “dudosas” motivaciones (y vinculaciones) que estarían detrás de estos reclamos.

Ante la inusitada repercusión de estos hechos, el "conflicto de Pulmarí” se transformaría en emblemático de la lucha del pueblo Mapuche y del conjunto de los grupos originarios de la Argentina. Por otro lado, como resultado de este conflicto, se recuperaron 42.000 hectáreas de su territorios ancestrales (Nawell et al. 2004).

Desde aquellos años la región se caracteriza por elevados niveles de conflictividad (con diferentes recuperaciones de campos reclamados por las comunidades, desalojos, etc.), la judicialización de la movilización indígena, así como por la persistencia de diversas irregularidades y fuertes controversias en cuanto a las concesiones otorgadas. A esto se le suman las disputas entre los variados sectores involucrados (indígenas, pobladores criollos, propietarios privados, empresas, la CIP, etc.) por los preciados recursos. En cuanto a la administración de la CIP, en los últimos años se ha dado cierto cambio de política en favor de las comunidades, fruto en gran medida de la movilización indígena.

\section{Las comunidades Mapuche, fuentes de ingreso y modos de reproducción social}

Las familias que actualmente conforman estas comunidades Mapuche del Departamento Aluminé, están organizadas a partir de diferentes troncos parentales sobrevivientes de la denominada “Campaña del Desierto”, que tuvo lugar a fines del Siglo XIX ${ }^{12}$. Sin embargo, la zona se encontraba 
poblada por diferentes parcialidades indígenas (como los huilliches) desde varios siglos antes de dicha avanzada militar.

Aluminé es, actualmente, uno de los Departamentos de la Provincia de Neuquén que posee mayor proporción de población indígena $(27,8 \%)^{13}$ muy superior al de la provincia en su conjunto (9,8\%) (INDEC 2001) $)^{14}$. En la actualidad, producto de nuevas reorganizaciones territoriales y adscripciones étnicas, son nueve las comunidades de este distrito, cinco de ellas en jurisdicción de la CIP. En total, conforman un núcleo poblacional que supera las 450 familias, equivalente a más de 2000 personas (Stecher y Berenger 2009), lo que representa un porcentaje muy significativo del total de la población del Departamento.

A esto se le debe adicionar los pobladores indígenas que residen como resultado de los procesos migratorios- en la localidad de Aluminé.

Previamente es necesario destacar que en Patagonia tienen una fuerte incidencia las formas de tenencia de la tierra como "ocupantes fiscales" (comúnmente denominados "fiscaleros") que se ubican en tierras que permanecen en manos de los Estados provinciales o nacional y de comunidades indígenas. Una variante de esta modalidad, es la que se registra en Pulmarí (para los diferentes sectores): se trata de ocupantes que -aunque se encuentran reconocidos por las autoridades oficiales- no cuentan con la propiedad como es el caso de los concesionarios y los “pobladores de ley” (quienes tenían residencia o derechos de pastaje en el área de Pulmarí previo a la conformación de la CIP) (Stecher y Berenger 2009). Esto creó una situación de gran inseguridad y a la vez fuertes conflictos cuando se trata de defender los territorios que ocupan y trabajan, frente al Estado, otros productores, empresas, etc.

En lo que respecta a las diferentes fuentes de ingreso de las familias indígenas, se encuentran tanto las de tipo extraprediales (aquellas generadas fuera del predio de la unidad doméstica) como aquellas prediales (dentro del mismo), siendo necesario comprenderlas de forma diferenciada y al mismo tiempo interrelacionadas.

Entre las primeras, en los casos menos frecuentes, los pobladores indígenas se desempeñan como trabajadores de planta permanente, mayormente en reparticiones del Estado nacional o provincial como instituciones escolares (auxiliares de servicios, maestros de huerta, de lengua Mapuche; etc.), en servicios de salud (agentes sanitarios), o como empleados de Parques Nacionales. Cabe destacar, el rol que desempeña CORFONE como demandante de empleo asalariado directo e indirecto en la región, en especial para los integrantes de las comunidades de la zona, que son convocados -ya sea por intermedio de contratistas o en forma directa por la empresa-. Las tareas realizadas son aquellas relacionadas con el manejo silvícola: plantación, poda y raleo. De acuerdo a la información recabada en las comunidades, el empleo temporario en estas labores resultaba ser el más significativo entre los integrantes masculinos, junto con las actividades relacionadas con el turismo y la construcción. 
Sin embargo, los trabajos asalariados más extendidos son los de carácter temporario que desarrollan los miembros de las familias en ámbitos rurales próximos a las comunidades, que requieren de una baja calificación y por ende, suelen ser menos remunerados, como la actividad de plantación, al igual que diversas tareas rurales (como el característico trabajo en las estancias). Dada la importancia regional del turismo, algunos integrantes de las comunidades logran desempeñarse en diversos empleos de este rubro, lo cual implica movimientos migratorios estacionales hacia las localidades de Aluminé y Villa Pehuenia -principalmente en la construcción en el caso de los hombres y servicio doméstico entre las mujeres-.

Además de los trabajos asalariados, otra fuente fundamental en los ingresos domésticos surge directamente del Estado, a través de diferentes beneficios sociales. Si bien estos aportes son legítimos desde la óptica de las necesidades de las comunidades, también es posible comprenderlos desde ciertas prácticas del clientelismo político, ya que generan un espiral de dependencia permanente.

Las políticas estatales de beneficios sociales, junto con la creciente escasez de trabajos asalariados en el medio urbano que se da a partir de la década de 1990 -como resultado de la aplicación de las políticas neoliberales, y su consiguiente incremento del desempleo y la precarización laboralconstituyen factores centrales a la hora de explicar la retención de esta población en el medio rural, en contraste con otras regiones y provincias. A este aspecto nos referiremos en el próximo punto, dada la importancia que poseen como fuente de ingreso.

En lo que respecta a las actividades prediales desarrolladas por las familias indígenas, la primera a destacar (por la importancia que posee) es la ganadería a nivel de subsistencia y/o para la comercialización (luego volveremos sobre este punto).

Finalmente, también se desarrollan otras actividades en el ámbito familiar o comunitario -algunas estrechamente relacionadas con prácticas culturales del Pueblo Mapuche- posibilitando así desplegar una pluriactividad y, por lo tanto, diversificar sus ingresos. Tal es el caso de la capitalización en ganado mayor (vacunos y equinos) que efectúan algunas familias -que luego veremos-, la confección y comercialización de artesanías (en lana y madera), la recolección de productos no maderables del bosque (piñones de araucaria, plantas medicinales, especies tintóreas, etc.), como así también la prestación de diversos servicios turísticos y la elaboración de ciertos productos para la venta, ligados a esta actividad (pan casero, dulces, etc.) (Stecher 2011).

\section{Políticas sociales y procesos de "reterritorializacion"}

Como hemos adelantado y aquí profundizaremos, una fuente de ingreso fundamental de las unidades domésticas Mapuche, surge directamen- 
te del Estado, a través de diferentes beneficios sociales. Para comprender esta característica, es necesario remitirnos a ciertas particularidades de la Provincia de Neuquén en su génesis y desarrollo histórico, lo que permite dar cuenta de su fuerte intervencionismo en materia socioeconómica, política y cultural.

Neuquén obtuvo el status de provincia en el año 1955 (luego de ser Territorio Nacional desde el año 1884) y las primeras elecciones de autoridades se efectuaron en 1958. Fue en 1963 que se conformó el partido provincial -el Movimiento Popular Neuquino (MPN)- a partir de figuras locales de amplio reconocimiento provenientes del peronismo.

Durante las décadas subsiguientes, la economía provincial se basó en la expansión del gasto público y en las rentas provistas por las empresas del Estado nacional -principalmente YPF (Yacimientos Petrolíferos Fiscales), Gas del Estado, etc. ${ }^{15}$-. Así es como se fue delineando el perfil productivo actual, basado en los recursos energéticos provenientes de la extracción gasífera, petrolífera e hidroeléctrica ${ }^{16}$.

En este contexto, el MPN consolidó su hegemonía política en estrecha relación con el desarrollo del Estado Provincial y el crecimiento de la economía regional, muy superior a la nacional. Ha desarrollado así -a lo largo de sus cinco décadas de existencia- una política que algunos autores han caracterizado como "una estrategia populista de desarrollo" (Favaro y Bucciarelli 2001) acorde con la poderosa estructura estatal de la provincia, lo que le ha permitido, a pesar de contar con diversos escenarios de crisis, gobernar la provincia desde su conformación como partido en todos los periodos constitucionales de esta etapa (años 1963-1966, 1973-1976 y 1983 hasta la actualidad). A esto se le debe sumar, que los ingresos derivados de las regalías ${ }^{17}$ hidrocarburíferas han crecido sensiblemente en los últimos años ante la suba en el precio internacional del crudo y los mayores volúmenes de exportación, lo que le ha permitido al estado provincial -y al partido de gobierno- contar con importantes recursos.

Así, es factible de comprender porque desde el momento de la provincialización, y luego acrecentado por esta matriz productiva -en conjunción con el afianzamiento del sistema político neuquino- el Estado provincial ha sido sumamente proactivo en la promoción de las áreas rurales. En este contexto, fueron efectuadas importantes obras de infraestructura como el tendido de rutas y caminos en sitios aislados, la creación de escuelas rurales con albergues, la implementación de un sistema de salud -con fuerte énfasis en la prevención y control sanitario- y diferentes planes de vivienda rural (Stecher y Lacalu 2010a).

Esta presencia estatal se tradujo en la temprana creación de "reservas” indígenas en el territorio provincial. En el año 1964, durante la primera gestión del gobernador Felipe Sapag ${ }^{18}$ (1963-1966), se llevó adelante el reconocimiento de diversas "reservas indígenas" en territorios ocupados por familias Mapuche ${ }^{19}$, por lo cual en la actualidad ascienden a 38 las 
comunidades formalizadas (Stecher 2011). No obstante, resultan 57 el número de agrupaciones reconocidas por la Confederación Mapuche Neuquina -organización indígena supracomunitaria- y que reclaman su institucionalización (ODHPI 2010).

Retornando a los beneficios sociales que reciben los integrantes de las comunidades del Estado, estos abarcan prácticamente a la totalidad de las familias. Están integrados por un componente monetario que promediaba los \$300 mensuales (a valores del año 2009 para las comunidades Currumil y Hiengheihual, en base a un estudio que efectuamos, Stecher y Berenger 2009), por el cual debe realizarse una tarea comunitaria como contraprestación. Para arribar a este cálculo, se consideró que cada unidad familiar es beneficiaria de al menos dos programas de empleo de \$150 cada uno, los que se perciben mensualmente durante todo el año.

A este ingreso se le suman los beneficios "no monetarios" que reciben de la Provincia de Neuquén, que consisten en la entrega de leña, garrafas de gas envasado (a través de un bono que implica un importante descuento) y caja alimentaria (una o dos por mes si la familia es numerosa), que también resultan -al efectuar su conversión a valores monetarios- aproximadamente otros \$200 mensuales (Ibid).

En lo que respecta a los ingresos resultantes de la ganadería, con el objetivo de cuantificar los valores que representan para cada unidad doméstica, retomamos la estimación que elaboramos en otra oportunidad (Stecher y Berenger 2009) a partir de la venta de animales en pie (ovinos y caprinos), de lana para el caso de los ovinos y pelo para los caprinos ${ }^{20}$. El cálculo por unidad doméstica, representa un ingreso que varía -según la comunidad- de \$200 mensuales a algo más de \$700 para las que poseen una situación más favorable. Desde ya es necesario considerar que estos valores representan promedios, con grandes niveles de diferenciación dentro de las mismas agrupaciones o entre las seis analizadas, plenamente comprensibles en función de heterogeneidades agroecológicas, así como efectos diferenciales de los procesos de intervención pública y de diversos proyectos de desarrollo ${ }^{21}$.

Ahora bien, si consideramos estas cifras para la ganadería -aún con las variaciones entre comunidades- mientras los ingresos de los subsidios monetarios, como vimos suman \$300 y los no monetarios \$200 (por unidad doméstica), concluimos la trascendencia que poseen los subsidios estatales como fuente de subsistencia de las familias Mapuche.

A esto le debemos sumar los planes de mejora en infraestructura y servicios rurales que financia y mantiene el Estado, que crean puestos de trabajo en estas agrupaciones: programas de vivienda, construcción y mantenimiento de caminos, electrificación, etc. Estos empleos asalariados temporarios (que dependen de los plazos de realización de estas obras) se suman a los de tipo permanente, que se efectivizan en diferentes organismos públicos localizados en ámbitos rurales. De hecho en todas las áreas 
rurales del Departamento Aluminé donde residen comunidades Mapuche, el estado provincial presta servicios básicos de salud y educación. En cada una de ellas, funciona al menos una escuela de nivel inicial y una posta sanitaria (Stecher y Berenger, 2009).

De allí que concluyamos la importancia del Estado -a través de diversos planes sociales, programas de infraestructura y puestos de trabajo permanentes- en la supervivencia de estas familias indígenas y en la retención de la población rural; pero también son nodales en la redefinición de las relaciones sociales en los ámbitos rurales (y también en la interacción con los urbanos).

\section{Pulmarí a contrapelo de la tendencia general: retención de la población rural, dinámicas regionales y locales}

Esta importancia de los planes sociales que hemos presentado en el apartado anterior, puede visualizarse en la retención de la población rural de este Departamento, por encima de la media provincial -y desde ya regional y nacional-.

Lo dicho no implica que no se produzcan migraciones rurales - urbanas, las que resultan características en la región, ante la imposibilidad por parte de los grupos domésticos indígenas de acrecentar las actividades económicas en la misma proporción que su crecimiento poblacional. Por eso, permanentemente las ciudades de la Patagonia reciben grandes contingentes de migrantes mapuches que se insertan en los circuitos productivos urbanos (Radovich y Balazote 2009). No obstante, estas migraciones son crecientemente de tipo estacional, tal como se viene destacando como una de las tendencias de los procesos rurales contemporáneos (Grammont 2008). De hecho, a partir del incremento de la actividad en la construcción, se han acrecentado los puestos asalariados en este rubro en el ámbito urbano -dada además la creciente importancia de Villa Pehuenia y Aluminé como centros turísticos-generando un nuevo movimiento migratorio pero de carácter estacional.

Este intervencionismo en el ámbito rural se puede observar tanto en la variación intercensal de la población rural para el Departamento Aluminé -considerando que una porción muy significativa corresponde a los integrantes de estas comunidades- como al visualizar la evolución poblacional a nivel de cada comunidad, donde resulta notorio el crecimiento demográfico de las agrupaciones formalizadas.

En el cuadro adjunto, presentamos los datos de evolución poblacional urbana y rural del total provincial y de los cuatro Departamentos del denominado “corredor de los lagos” de la Provincia de Neuquén (ver Mapa $\mathrm{N}^{\circ}$ 1). También adjuntamos los datos totales de la vecina Provincia de Río Negro y los del Departamento Bariloche, lindante con el de "Los Lagos" de Neuquén y que también integra esta región de los lagos. 


\section{Cuadro $N^{\circ}$ 1: Evolución de la población urbana, rural y total por Departamento del "corredor de los Lagos de las Provincias de Neuquén y Río Negro y totales por provincia}

\begin{tabular}{|c|c|c|c|c|c|}
\hline & \multirow{2}{*}{\begin{tabular}{|c|}
$1980(*)$ \\
Valores \\
absolutos
\end{tabular}} & \multicolumn{2}{|c|}{$1991\left(^{*}\right)$} & \multicolumn{2}{|c|}{$2001(* *)$} \\
\hline & & $\begin{array}{c}\text { Valores } \\
\text { absolutos }\end{array}$ & $\begin{array}{c}\text { Variac. } \\
1980-1991\end{array}$ & $\begin{array}{c}\text { Valores } \\
\text { absolutos }\end{array}$ & $\begin{array}{c}\text { Variac. } \\
1991-2001\end{array}$ \\
\hline TOTAL Neuquén & 243.850 & 388.833 & $59,50 \%$ & 474.155 & $21,94 \%$ \\
\hline Urbana & 185.608 & 335.553 & $81,10 \%$ & 419.983 & $25,16 \%$ \\
\hline Rural & 58.242 & 53.280 & $-9,60 \%$ & 54.172 & $1,67 \%$ \\
\hline Aluminé & 3.842 & 4.946 & $28,70 \%$ & 6.308 & $27,54 \%$ \\
\hline Urbana & 1.640 & 2.537 & $54,70 \%$ & 3.461 & $36,42 \%$ \\
\hline Rural & 2.202 & 2.409 & $9,40 \%$ & 2.847 & $18,18 \%$ \\
\hline Huiliches & 7.550 & 9.679 & $28,20 \%$ & 12.700 & $31,21 \%$ \\
\hline Urbana & 5.679 & 7.333 & $29,10 \%$ & 10.302 & $40,49 \%$ \\
\hline Rural & 1.871 & 2.346 & $25,40 \%$ & 2.398 & $2,22 \%$ \\
\hline Lácar & 14.193 & 17.085 & $20,40 \%$ & 24.670 & $44,40 \%$ \\
\hline Urbana & 12.239 & 14.842 & $23,70 \%$ & 22.432 & $51,14 \%$ \\
\hline Rural & 1.954 & 2.243 & $-0,30 \%$ & 2.238 & $-0,22 \%$ \\
\hline Los Lagos & 2.566 & 4.181 & $62,90 \%$ & 8.654 & $106,98 \%$ \\
\hline Urbana & 1.759 & 3.056 & $73,70 \%$ & 7.730 & $152,95 \%$ \\
\hline Rural & 807 & 956 & $39,40 \%$ & 924 & $-3,35 \%$ \\
\hline TOTAL Río Negro & 383.354 & 506.772 & $32,2 \%$ & 552.822 & $9,1 \%$ \\
\hline Urbana & 275.373 & 441.053 & $60,2 \%$ & 502.760 & $14,0 \%$ \\
\hline Rural & 107.981 & 65.719 & $-39,1 \%$ & 50.062 & $-23,8 \%$ \\
\hline Bariloche & 60.334 & 94.640 & $56,9 \%$ & 109.826 & $16,0 \%$ \\
\hline Urbana & 53.990 & 89.848 & $66,4 \%$ & 105.779 & $17,7 \%$ \\
\hline Rural & 6.344 & 4.792 & $-24,5 \%$ & 4.047 & $-15,5 \%$ \\
\hline
\end{tabular}

Fuente: $(*)$ = INDEC, 1991; (**) INDEC, 2001.

Nota: En el Departamento Aluminé la población urbana corresponde a la ciudad de Aluminé, en Huiliches pertenece a la localidad de Junín de los Andes, en el Departamento Lácar a San Martín de los Andes, en Los Lagos a Villa la Angostura y en Bariloche a las ciudades de San Carlos de Bariloche y el Bolsón.

Si consideramos - como forma de ver más claramente estos cambios-el veintenio 1980-2001, mientras la población rural en el total de la Provincia de Neuquén disminuyó un 7\%, la de Aluminé creció un 29,3\%, en términos comparables a Huiliches $(28,2 \%)$-que también posee comunidades reconocidas con una fuerte incidencia demográfica en el ámbito rural y similares situacio- 
nes en términos de intervencionismo estatal- (INDEC, 2001).

En la vecina provincia de Río Negro, con una política muy diferente en materia de promoción rural -y un escenario de crisis económica producto de la baja en los precios del ganado ovino- se observa en el conjunto de la Provincia y en el caso del Departamento Bariloche una baja incesante de la población asentada en el campo: en el total provincial la población rural decreció entre 1980 y 2001 un 53,6\% y en Bariloche un 36,2\%, por lo que el contraste con los departamentos neuquinos -en especial Aluminé y Huiliches- resulta altanamente relevante.

Esta misma tendencia, puede ser visualizada no ya a nivel censal sino en relación a cada comunidad, resultando sumamente ilustrativas las tendencias que estamos analizando, que surgen de la comparación en la cantidad de familias que integran estas seis comunidades entre los años 1998 y 2007.

\section{Cuadro No 2: Evolución del número de familias en comunidades del área CIP}

\begin{tabular}{|l|c|c|}
\hline Comunidad & $\begin{array}{c}\mathrm{N}^{\circ} \text { de familias año } \\
1998(*)\end{array}$ & $\mathrm{N}^{\circ}$ de familias año 2007 (**) \\
\hline Comunidad Currumil & 22 & 45 \\
\hline Comunidad Catalan & 44 & 82 \\
\hline Comunidad Puel & 45 & 202 \\
\hline Comunidad Aigo & 125 & 25 \\
\hline Norquinco & 23 & 60 \\
\hline Comunidad Hiengheihual (Salazar) & 27 & 459 \\
\hline TOTAL & 286 & 81 \\
\hline
\end{tabular}

Fuente:

(*) Universidad Nac. Del Comahue-INTA, 1999.

(**) Confederación Mapuce Neuquina, 2007.

Como es factible observar, se destaca un considerable incremento en el número de familias en el transcurso de este decenio, en que el registro asciende de 289 a 459 familias, es decir un aumento del 60,5\%. Indudablemente, esta tendencia es diametralmente diferente con lo que sucede con otras regiones con pequeños productores rurales, en las que se advierte un agudo proceso de “descampesinización” y “urbanización”. Este dato revelaría una dinámica que conlleva la permanente reconfiguración del uso del suelo por parte de las comunidades, cuyos límites obviamente son fijos -a diferencia de la dotación de los recursos agroecológicos necesarios para sostener dicho crecimiento poblacional-.

Los motivos de este fenómeno ya los hemos mencionado: surgen de la intervención directa del Estado a través de la aplicación de diversos sub- 
sidios (monetarios y no monetarios), planes de vivienda y prestación de servicios esenciales, etc.

Por otra parte, el proceso de conformación de nuevas unidades domésticas surge al consolidarse nuevas familias, las que solicitan a las autoridades de su comunidad una porción de tierra donde establecerse. Generalmente, como consecuencia de la imposibilidad de subdividir aún más el territorio, se les asignan parcelas en los lugares más pobres -entre los ya de por sí marginales-. De esta manera la única posibilidad de inicio productivo de las nuevas poblaciones, es utilizando ganado apto para estas áreas y de bajo costo de manejo, como la cría de ovinos, pero -fundamentalmentecaprinos (Stecher 2011).

\section{Los efectos de las políticas sociales en las actividades tradicionales: el caso de la ganadería}

Uno de los aspectos que deseamos destacar en función de los objetivos del presente, es visualizar la tenencia de ganado en las diferentes comunidades y la evolución en los años 1998, 2003 y 2007. Cabe señalar que el 82\% del total del stock ganadero existente en Pulmarí, pertenece a productores de las comunidades Mapuche (López Espinoza 2008). Como puede apreciarse en el cuadro $\mathrm{N}^{\circ} 3$ para cada tipo de ganado en el conjunto de las comunidades en estos tres momentos, se observa una tendencia al crecimiento en la cantidad de cabezas. Lo mismo se puede observar a nivel de cada comunidad, si bien existen algunas situaciones específicas que llevan en ciertos casos a una baja. Tal el caso de Currumil, con una disminución en el número de cabezas de bovinos y caprinos - dada la imposibilidad de contar con tierras aptas-, una baja en Ñorquinco (en bovinos) y en Hiengheihual (en bovinos y muy levemente en ovinos), variaciones que a veces se explican por la transferencia hacia otro tipo de ganado. No obstante, la tendencia general es marcadamente hacia la suba.

\section{Cuadro No 3: Evolución de la cantidad de Ganado por tipo y comuni- dad que pastorean en el Departamento Aluminé}

\begin{tabular}{|c|c|c|c|c|c|c|c|c|c|}
\hline & \multicolumn{3}{|c|}{ Bovinos } & \multicolumn{3}{|c|}{ Ovinos } & \multicolumn{3}{|c|}{ Caprinos } \\
\hline & $\frac{\circ}{g}$ & 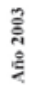 & 商 & $\frac{\stackrel{\infty}{\alpha}}{g}$ & 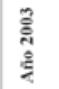 & 䒽 & $\frac{\stackrel{\infty}{\mathrm{O}}}{\stackrel{8}{g}}$ & 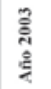 & 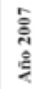 \\
\hline \multicolumn{10}{|l|}{ Comunidad } \\
\hline Puel & 933 & 850 & 1.000 & 632 & 338 & 279 & 1.752 & 493 & 309 \\
\hline Catalän & 335 & 477 & 517 & 2.450 & 4436 & 7.664 & 3.114 & 3570 & 4167 \\
\hline Norguinco & 410 & 265 & 194 & 1.388 & 751 & 1.095 & - & 10 & 25 \\
\hline $\begin{array}{l}\text { Hiengheihual } \\
\text { (Salazar) }\end{array}$ & 540 & 384 & 353 & 2.923 & 2815 & 2.704 & 780 & 840 & 992 \\
\hline Aigo & 968 & 1135 & 1.815 & 6.013 & 18.933 & 21.291 & 3.473 & 5.234 & 6.777 \\
\hline Curnumil & 192 & 151 & 118 & 809 & 1.103 & 1.305 & 980 & 703 & 570 \\
\hline
\end{tabular}

Fuente: López Espinoza, 2008. 
El caso de la comunidad Puel, ilustra las heterogeneidades intra e intercomunitarias, su incidencia en la dinámica productiva y en el uso de los territorios. Algunos integrantes de esta comunidad que desarrollan actividades ganaderas, se encuentran en una fase de capitalización centrada en el aumento del stock de bovinos y -concomitantemente- la disminución de ganado menor, contraponiéndose a la tendencia hacia el aumento de ovinos y caprinos de las restantes agrupaciones. Este proceso es explicable gracias a los beneficios obtenidos por aquellos que se integraron al modelo de desarrollo implementado desde el Estado provincial, que realiza una actividad forestal sustentada en el trabajo asalariado de algunos integrantes de la comunidad $^{22}$.

Lo aquí ejemplificado, permite visualizar como a partir de una activa política pública como es la promoción de la actividad forestal, se generan profundas transformaciones en las actividades caracterizadas como "tradicionales” como es la ganadería, con efectos en el conjunto de las unidades domésticas y en el acceso y el uso del territorio.

\section{Ruralidad, paradojas y tensiones asociadas a la movilización del pueblo Mapuche en Pulmarí: final abierto}

A partir de las dinámicas analizadas para la población Mapuche de Pulmarí, observamos cómo la población rural que integra las comunidades, viene experimentando una profunda transformación, producto de una multiplicidad de factores diferenciados e interrelacionados y que influyen en diferentes niveles. En algún sentido, respondiendo así a la pregunta disparadora de este dossier temático, entendemos que lejos está de "extinguirse" el campesinado o el ámbito de lo "rural", sino en todo caso se encuentra experimentando profundas transformaciones, las que por su multidimensionalidad y complejidad, apenas comenzamos a apreciar -al menos para esta región, luego de años de trabajo- en toda su magnitud.

En este sentido, el rol de la provincia de Neuquén, su fuerte política intervencionista en la sociedad neuquina en general y en particular en los ámbitos rurales y la disponibilidad de recursos provenientes de la renta petrolera, resulta una variable fundamental para explicar las dinámicas de dichas transformaciones. Esto refuerza nuestro planteo de un marco temporal y territorial más amplio para el abordaje de las poblaciones rurales, de allí los aportes de los denominados enfoques "histórico-regionales" para abordar estas variables.

No obstante los pobladores, sobre la base de esa realidad (que es también en gran medida resultante de las acciones que han efectuado como colectivo social) despliegan sus estrategias que generan profundos cambios.

En este sentido, tenemos que considerar no sólo las implicancias productivas, sino también las construcciones identitarias que se dan entre 
los integrantes de las comunidades Mapuche, en relación al territorio, vinculándolas a la existencia del grupo mismo (Bartolomé 1997; Barabas 2004). Tal como lo plantea Alicia Barabas, quién concibe al “(...) territorio como espacio culturalmente construido por la sociedad" (2004:112), donde los denominados "etnoterritorios" -tal como los define esta autora- no solo proveen: “(...) la reproducción física de la población sino que en él se desarrollan relaciones de parentesco, culturales, lingüísticas y políticas. El etnoterritorio reúne las categorías de tiempo y espacio (historia en el lugar) y es soporte central de la identidad y la cultura" (2004:112-113). En relación a este último aspecto, entendemos una de las acciones desarrolladas por los pobladores, como es la tenencia de ganado que -a pesar de la falta de tierras- sigue en ascenso. El mismo constituye una fuente de subsistencia, pero es también un recurso muy asociado al Mapuche en términos identitarios, símbolo de prestigio y diferenciación social. Actualmente, existe una tendencia a incrementar la tenencia de caprinos, hecho explicable ya que el mismo se adapta a tierras productivamente marginales (mientras los otros tipos de ganado poseen mayores requerimientos pastoriles). Por otro lado, la única forma, por parte de una familia campesina de contar con espacios para pastoreo, es consolidarse en su rol de crianceros teniendo existencias ganaderas, lo que genera una retroalimentación donde la posesión de ganado es la forma de acceder a los ámbitos de pastoreo, de allí la tendencia a incrementarlo. Por otro lado, las existencias ganaderas claramente no resultan suficientes para que las unidades domésticas centralicen su fuente de supervivencia en el recurso ganadero, pero fomentan el incremento del mismo (siempre en la medida de sus posibilidades). A la vez las familias, buscan paralelamente, estrategias para diversificar sus ingresos.

Lo mismo podemos decir en relación al ejemplo que dimos de la capitalización de ganado bovino en la comunidad Puel, lo que entendemos resulta por demás paradigmático de cómo la pluriactividad -resultante de la forma en que se han ido complejizando las relaciones en el campo- ha redefinido el conjunto de las relaciones sociales, llegando incluso a modificar profundamente las actividades consideradas "tradicionales" (como la ganadería). Es necesario aquí remarcar, como parte de este complejo entramado -tal como lo han señalado Tiscorna (2007) y López Espinosa (2008) para estas comunidades de Pulmarí- la gran heterogeneidad social dentro de las mismas comunidades, muchas veces soslayada desde la noción predominante que existe sobre los pueblos indígenas y, también desde las mismas políticas de intervención.

En este sentido, debemos considerar cómo en la memoria colectiva de la población indígena existe una clara noción del carácter ancestral de estos territorios en que se encuentran asentados, que fueron primero apropiados durante la "Conquista del Desierto". Posteriormente, se establecieron las diferentes instituciones con importantes consecuencias (Ejército, Parques Nacionales) y finalmente, fue impuesta la jurisdicción de la CIP que agravó las condiciones de vida de estas poblaciones y conllevó la presencia de diferentes actores (como los concesionarios), fuertemente enfren- 
tados con las comunidades indígenas, si bien en los últimos años y como resultado de la movilización indígena, esta situación se está revirtiendo.

Estas tendencias aquí descriptas, que implican una "territorialización” o "re-territorialización”, conllevan una serie de crecientes litigios hacia el interior de las propias comunidades y hacia afuera de las mismas, lo que entendemos- constituye una de las causas de la conflictividad que se registra desde hace dos décadas en la región de Pulmarí 


\title{
Nota
}

\begin{abstract}
${ }^{1}$ Este trabajo se enmarca en el proyecto UBACyT 2012-2015: “Etnicidades, movimientos y comunidades indígenas en contextos de promoción de emprendimientos productivos: una perspectiva comparativa entre los pueblos indígenas Mapuche, Chané, y Qom”, Facultad de Filosofía y Letras, Universidad de Buenos Aires, bajo la dirección del Dr. Sebastián Valverde.
\end{abstract}

\begin{abstract}
${ }^{2}$ Mapu significa en "mapudungún” (en su lengua originaria) “tierra” y “che” significa "gente”, así “Mapuche” quiere decir “Gente de la tierra”. Este pueblo indígena que se asienta en el sur de Chile y de Argentina (en el área norte de la Patagonia), sobrevivió a los ataques genocidas y etnocidas llevados a cabo a ambos lados de la cordillera de los Andes a fines del Siglo XIX (Radovich y Balazote 2009). En el vecino país de Chile se asientan en la Octava, Novena y Décima Región y (como resultado de las migraciones) en la región Metropolitana, sumando -de acuerdo a lo que señala Bengoa (2007)- 900.000 integrantes, de los cuales 250.000 habitan en el campo y el resto en las ciudades. En Argentina, se asientan en las provincias de Chubut, Río Negro, Neuquén, La Pampa y Buenos Aires (Radovich y Balazote 2009) conformando algo más de 200.000 miembros de acuerdo al último censo de población del año 2010 (INDEC 2010), siendo el pueblo originario más numeroso del país (seguido por los grupos Qom-Toba, Guaraní, Diaguita y Kolla).
\end{abstract}

${ }^{3}$ El directorio de la CIP está compuesto por ocho miembros, cuatro del Estado nacional, tres de la provincia de Neuquén y un representante indígena.

${ }^{4}$ La antigua estancia Pulmarí, fue expropiada a la familia Miles (de capitales ingleses) a fines de los años '40 durante la presencia de Juan D. Parón, y cedida al Ejército Argentino.

${ }^{5}$ Empresa que también se puede caracterizar como "para-estatal” (Stecher y Laclau 2010a). Creada en el año 1974, está integrada por capitales mayoritarios del Estado Provincial y un sector de inversión privada. Para una caracterización de CORFONE ver Laclau y Stecher (2010a) y (2010b) y también lo que abordamos en otro artículo (Stecher y Valverde 2012) en relación a los conflictos territoriales asociados a la explotación forestal.

${ }^{6}$ En otro artículo (Valverde, en prensa 2013) analizamos específicamente el proceso de convergencia disciplinar entre la Historia y la Antropología desarrollado en el ámbito académico argentino, y la potencialidad del uso de diferentes categorías y lecturas de los "enfoques histórico-regionales” para el abordaje de los movimientos indígenas.

${ }^{7}$ Esto es explicable ya que en la ciudad de Neuquén (capital de la Provincia homónima) desde hace varios años se dicta la carrera de Historia en la Universidad Nacional de Comahue y hay diversos grupos consolidados de investigadores, situación que difiere de otras carreras afines (Sociología, Antropología, etc.), cuyo dictado en la zona es mucho más reciente, y el desarrollo de equipos profesionales es incipiente. Esta situación permite entender la influencia de la disciplina histórica sobre las restantes.

${ }^{8}$ Villa Pehuenia, se encuentra ubicada a $302 \mathrm{~km}$ de la ciudad de Neuquén, en una zona de grandes bellezas naturales, en la margen norte del lago Aluminé, contando con una población aproximada de 900 habitantes. Se inició como un asentamiento de segundas residencias de uso turístico, siendo su principal actividad económica el turismo -tanto estival como invernal- (Stecher y Berenger 2009). 
${ }^{9}$ Censo Nacional de Población, Hogares y Viviendas 2001, Instituto Nacional de Estadística y Censos (INDEC). Reprocesamiento propio de la Base de datos "Redatam+SP" disponible en www.indec.gov.ar

${ }^{10}$ La problemática de la actividad forestal en relación a las comunidades Mapuche de la zona, la abordamos específicamente en otro artículo (Stecher y Valverde 2012).

${ }^{11}$ Decreto No 1.410 del 25/8/1987, ratificado por Ley No 23.612 de 1988 (Radovich 2000).

${ }^{12}$ De hecho, en esta región se llevó a cabo en los últimos meses del año 1882 y principios de 1883 una de sus batallas, la denominada "Campaña de los Andes”. El objetivo final de la misma consistió en ocupar los puntos estratégicos sobre la cordillera, quedando definitivamente conquistado el territorio del Neuquén, y someter los últimos grupos Mapuche que aún no se habían subordinado a las fuerzas nacionales (Tato Vázquez 2011), entre los que se incluyen las agrupaciones de Reuquecurá y Namuncurá.

${ }^{13}$ Estos datos deben ser considerados a modo de señales ya que no discriminan los diversos grupos indígenas, y a la vez están incluidos aquí quienes se reconocen indígenas que son migrantes de otras regiones o países. No obstante, es plenamente utilizable como indicador a modo de tendencia, ya que la gran mayoría se trata de los integrantes del pueblo Mapuche oriundos de esta zona.

${ }^{14}$ Tomamos como referencia los datos del año 2001 ya que los del año 2010 no se encuentran aún desagregados en estos indicadores.

${ }^{15}$ En el año 1918 se descubrió la existencia de petróleo en la provincia (Favaro y Bucciarelli 2001) y con la creación de YPF -en el año 1922- se fue promoviendo la transformación de la base productiva de la provincia.

${ }^{16}$ La provincia de Neuquén produce, en base a valores del año 2010, 7,8 millones de m3 de petróleo (que representan el 23\% de la producción de la Argentina), 22,4 miles de millones de $\mathrm{m} 3$ de gas (48,1\% del total del país), siendo en estos indicadores la primera provincia del país en producción (Provincia del Neuquén - Dirección Provincial de Estadística y Censos, 2011a). En cuanto a su participación en el Producto Bruto Geográfico (PBG) este rubro (“Explotación de Minas y Canteras”) fue para el año 2008 del 47,6\% del total del presupuesto de la provincia a valores constantes 1993/2008 (Provincia del Neuquén - Dirección Provincial de Estadística y Censos, 2011b).

${ }^{17}$ La regalía es la apropiación de la renta hidrocarburífera por parte de la provincia poseedora del recurso, gravitando significativamente en los ingresos provinciales. En el caso de Neuquén llegó a representar en 1988 más del 50\% del presupuesto (Favaro y Bucciarelli 2001). En la actualidad la provincia cobra el $21 \%$ de las regalías de Petróleo del país y el $59 \%$ de las de Gas (en términos muy similares a los volúmenes de producción), y han crecido sensiblemente en la última década, fundamentalmente ante el alza de los precios internacionales (Secretaría de Política Económica - Subsecretaría de Programación Económica, 2011).

${ }^{18}$ Felipe Sapag fue el fundador e histórico líder del Movimiento Popular Neuquino (MPN), y cuatro veces gobernador de la provincia.

${ }^{19}$ Para poder materializar la entrega de títulos de propiedad resultaba indispensable darle a 
las comunidades alguna forma de organización. Por ello desde el gobierno provincial se promovió la figura de la Asociación de Fomento Rural (AFR) y la asociación civil como de efectivizar dicha regularización.

${ }^{20}$ Para la estimación de estos ingresos resultantes del ganado ovino y caprino, se consideró un porcentaje de comercialización del 25\% del stock ganadero de cada tipo de animal al año, a un valor de $\$ 180$ por cabeza. A eso se le suman los ingresos por la venta de lana (para el caso de los ovinos, $3 \mathrm{Kg}$. por cabeza a $\$ 9$ el Kg, siempre a valores del año 2009) y de pelo para los caprinos (1,3 Kg. por cabeza a un valor de \$3 el Kg) (Stecher y Berenger 2009). No contabilizamos ingresos por venta de vacunos debido a que la tendencia registrada en la zona es a incrementar el número de cabezas (proceso de capitalización) y al consumo doméstico, no a la comercialización.

${ }^{21}$ A modo de ejemplo de estas variabilidades, mientras en Puel el 63,0\% de sus existencias de ganado son vacunos, $17,6 \%$ de Ovinos y 19,5\% de Caprinos (en base a valores del año 2007) en Catalán la distribución es de 4,2\% bovinos, 62,1\% de ovinos y el 33,7\% son Caprinos (López Espinosa 2008). Luego explicaremos las causas de esta presencia de bovinos en Puel, como ejemplo de los procesos de diferenciación, en gran medida resultado de las políticas públicas.

${ }^{22}$ En el año 1975, en el marco de las políticas aplicadas por la Provincia, se incorporaron como asalariados de planta miembros de las comunidades Puel, y en menor medida Catalán. En el primer caso (Puel), los productores ingresaron capacitándose como operarios del vivero, de manera permanente, desde los inicios mismos del proyecto. Es decir, tanto el vivero como el empleo público como política forestal, resultaron los factores centrales para explicar el desarrollo de esta actividad en dicha comunidad. Esta metodología no se repitió de igual manera en otros casos de la zona, lo que contribuye a explicar los niveles diferenciales de adopción de las actividades de forestación (Stecher 2011; Stecher y Valverde 2012). En la actualidad, como resultado de esta activa política provincial, 713 hectáreas de la comunidad Puel se encuentran forestadas (sobre un territorio de 11.959) lo que representa el 5,96\% de la superficie de su territorio (cuando ninguna otra agrupación, supera el 1,5\%) (López Espinosa 2008). 


\section{Bibliografía}

Bandieri, Susana (2001), “Haciendo Historia regional”. En: Pueblos y Fronteras de la Patagonia Andina. Revista de Ciencias Sociales. 2, El Bolsón, Río Negro, Argentina.

Barabas, Alicia (2004), "La territorialidad simbólica y los derechos territoriales indígenas: reflexiones para el estado pluriétnico”. En: Alteridades, 14, 27. Universidad Autónoma Metropolitana - Iztapalapa, México.

Bartolomé, Miguel (1997), Gente de costumbre y gente de razón. Las identidades étnicas en México. Siglo XXI Editores, México DF.

Bengoa, José (2007), La Emergencia Indígena en América Latina. Fondo de Cultura Económica, Santiago de Chile.

Carrasco, Morita; Briones, Claudia (1996), "Pulmarí. La esperanza mapuche bajo acoso judicial”. En: La tierra que nos quitaron. Reclamos indígenas en Argentina. Copenhague. Documento IWGIA, Copenhague.

Confederación Mapuce Neuquina (2007), Relevamiento Técnico, Jurídico y Catastral de las Comunidades Mapuche en la Provincia de Neuquén. Instituto Nacional de Asuntos Indígenas, Neuquén.

Corporación Interestadual Pulmarí (2012), Sitio web institucional. http:// www.pulmari.org/index.php? option=com_content\&view $=$ article $\&$ id $=46 \&$ Itemid $=59$

Delrio, Walter; Lenton, Diana; Papazian, Alexis (2010), “Agencia y política en tres conflictos sobre territorio Mapuche: Pulmarí / Santa Rosa-Leleque / Lonko Purrán”. En: Sociedades de Paisajes áridos y semi-áridos. Revista Científica del Laboratorio de Arqueología y Etnohistoria de la Facultad de Ciencias Humanas, vol. II, Universidad Nacional de Río Cuarto, Córdoba.

De Grammont, Hubert (2008), “El concepto de nueva ruralidad”. Pérez Correa, Edelmira; Farah Quijano, María Adelaida, Hubert Carton de Grammont (compliadores). La nueva ruralidad en América Latina - Avances teóricos y evidencias empíricas. Editorial Pontificia Universidad Javeriana: Consejo Latinoamericano de Ciencias Sociales, Bogotá.

Giarraca, Norma (2001), “Prologo”. En: Norma Giarracca (comp.) ¿Una nueva ruralidad en América Latina? CLACSO, Buenos Aires.

Favaro, Orietta y Bucciarelli, Mario (2001), "Reflexiones en torno a una experiencia populista provincial. Neuquén (Argentina), 1960-1990”. Nueva Sociedad. Nro. 172. Marzo / Abril 2001, Caracas, Venezuela.

INDEC - Instituto Nacional de Estadísticas y Censos (2010), Censo Na- 
cional de Población, Hogares y Viviendas, 2010. Buenos Aires, Argentina. En: <http://www.indec.gov.ar>

Ídem (2001), Censo Nacional de Población, Hogares y Viviendas, 2001. Buenos Aires, Argentina. En: http://www.indec.gov.ar/

Ídem (1991), Censo Nacional de Población y Vivienda, 1991. Buenos Aires, Argentina.

López Espinosa, Fernando (2008), Aptitud forrajera y estimación de la capacidad de pastoreo de la Corporacion Interestadual Pulmarí y comunidades mapuche aledañas. Informe interno CIP. Neuquén: Aluminé.

Nawel, Xalkan Wenu; Huerco, Luisa; Loncon, Lorenzo; Villarreal, Jorgelina (2004), "Pulmarí: recuperación de espacios territoriales y marco jurídico: desafíos mapuches a la política indigenista del Estado. Informe de caso Proyecto 'Desarrollo Comunitario en Perspectiva Comparada'”, en: Centro de Política Social para América Latina (CLASPO), Universidad de Texas, EE.UU.

Ñancucheo, Jorge (1998), "Pulmarí en la lucha del pueblo mapuche”. Nosotros los otros, año 2, n. 3. Facultad de Filosofía y Letras, UBA, Buenos Aires.

ODHPI - Observatorio de Derechos Humanos de Pueblos Indígenas (2010), Informe de Situación de los Derechos Humanos del Pueblo Mapuce en la Provincia del Neuquén, 2009-2010, Neuquén Argentina.

Provincia del Neuquén - Dirección provincial de Estadística y Censos de la Provincia del Neuquén (2011a). Anuario Estadístico de la Provincia del Neuquén, Año 2011, Neuquén, Argentina.

Ídem (2011b), Informe Producto Bruto Geográfico - Años 1993/2009, Neuquén, Argentina.

Radovich, Juan Carlos (2000), “Identidad y conflicto en territorio mapuche: el caso Pulmarí”. En: $50^{\circ}$ Congreso Internacional de Americanistas. Varsovia, Polonia, 10 al 14 de julio de 2000.

Radovich, Juan Carlos; Balazote, Alejandro (2009), "El pueblo mapuche contra la discriminación y el etnocidio”. En: Ghioldi, Gerardo (Comp.). Historia de las familias mapuche Lof Paichil Antriao y Lof Quintriqueo de la margen Norte del lago Nahuel Huapi. Archivos del Sur, Villa la Angostura, Argentina.

Roseberry, William (1991), “Los campesinos y el mundo”. En: Stuart Plattner (ed.) Antropología Económica. CONACULTA - Consejo Nacional para la Cultura y las Artes/ Alianza Editorial, Ciudad de México. 
Rubio, Blanca (2002), “La exclusión de los campesinos y las nuevas corrientes teóricas de interpretación”, Nueva Sociedad, 182, Caracas.

Secretaría de Política Económica - Subsecretaría de Programación Económica (2011), “Complejo Petróleo y Gas” - Serie "Producción Regional por Complejos Productivos”.

Stecher, Gabriel (2011), Territorio, Desarrollo e intervenciones institucionales en comunidades mapuce Los casos del Área Pulmarí (Departamento Aluminé), Linares (Departamento Huiliches) y Vera (Departamento Lacar) Provincia de Neuquén. Tesis de Doctorado en Estudios Sociales Agrarios Centro de Estudios Avanzados - Facultad de Ciencias Agropecuarias Universidad Nacional de Córdoba, Córdoba.

Stecher, Gabriel; Berenger, Paula (2009), Estudio de impacto socioeconómico en territorios de las comunidades mapuce Currumil, Aigo y Catalan. Proyecto de Fortalecimiento del $M D L$ (F/R) en la República Argentina. Documento técnico. JICA-MRI.

Stecher, Gabriel; Lacalu, Pablo (2010a), “Desarrollo forestal y conflictos inter-étnicos en Aluminé, Neuquén, Argentina”. En: Congreso Latinoamericano de Sociología Rural, 8. Porto de Galinhas (Recife-Brasil), 15 a 19 de Noviembre de 2010.

Ídem (2010b), “Interfases fronterizas y disputas inter-étnicas. La conflictividad recurrente en Aluminé, Neuquén. En: $7^{\circ}$ Congreso Chileno de Antropología, San Pedro de Atacama, Chile, 25-29 de Octubre de 2010.

Stecher, Gabriel; Valverde, Sebastián (2012), “Los proyectos de desarrollo rural y forestal en contextos de pluriculturalidad. Las comunidades indígenas en la jurisdicción de la 'Corporación Interestadual Pulmarí', Provincia de Neuquén, Argentina”. En: INTERAÇÕES - Revista Internacional de Desenvolvimento Local. Vol. 13. N² 2, Julio/Diciembre 2012. Campo Grande, Mato Grosso do Sul, Brasil.

Tato Vázquez, Paula (2011), “Capítulo II: primeros antecedentes históricos sobre Ñorquinco y su población”. En: Valverde, Sebastián, Crosa, Zuleika, Gónzalez Palominos, Karina y Tato Vázquez, Paula (2011), Los pobladores del Lof Ñorquinco: de la expulsión a la reconstrucción. Facultad de Filosofía y Letras, Universidad de Buenos Aires, Buenos Aires.

Teubal, Miguel (2001), “Globalización y nueva ruralidad en América Latina”. En: Norma Giarraca (comp.) ¿Una nueva ruralidad en América Latina? Buenos Aires: CLACSO, Buenos Aires.

Tiscornia, Luis (2007), “La caracterización social de los pueblos originarios. Las comunidades mapuce en el área Pulmarí de la provincia de Neuquén”. En: V Jornadas Interdisciplinarias de Estudios Agrarios y Agroindustriales. Facultad de Ciencias Económicas de la Universidad de 
Buenos Aires, 7, 8 y 9 de noviembre de 2007.

Universidad Nacional del Comahue - I.N.T.A. (1999), Propuesta para el uso, aprovechamiento y manejo integral de los campos Pulmari y Comunidades Indígenas, Aigo, Salazar, Currumil, Catalan, Puel y Ñorquinco. Bariloche, Río Negro.

Valverde, Sebastián (2009), “Identidad étnica, etnicidad y reorganización comunitaria: el caso de la agrupación Mapuche Ñorquinco (provincia de Neuquén)”. № 17, Pap. trab. - Cent. Estud. Interdiscip. Etnollingüíst. Antropol. Sociocult, Rosario.

Valverde, Sebastián (2013 en prensa), “Convergencias disciplinares entre Historia y Antropología: la perspectiva 'histórico-regional’ y las investigaciones sobre los pueblos indígenas en Norpatagonia Argentina”. En: Revista Internacional de Ciencias Sociales interdisciplinarias (En prensa, Volumen 2, Número 1, 2013) Common Ground Publishing. Madrid, España.

Wolf, Eric (1993), Europa y la gente sin historia. Fondo de cultura económica, México.

Recibido: 14.02.2013

Aceptado: 07.03.2013 